
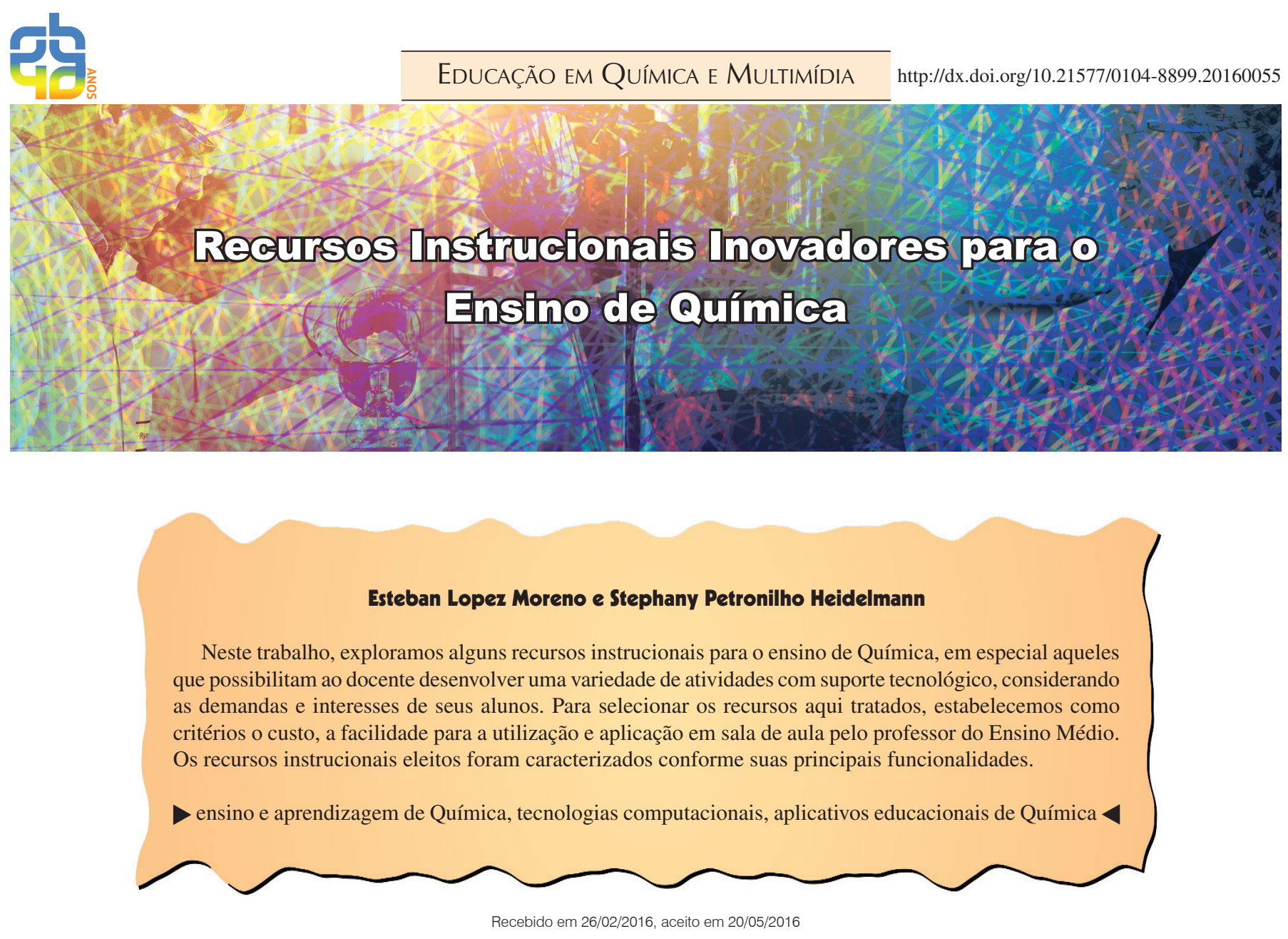

E m termos tecnológicos, nossa sociedade mudou substancialmente nas últimas décadas. Não apenas os computadores passaram a fazer parte, de maneira quase onipresente, do espaço escolar ou das residências, como também os smartphones invadiram as mãos de alunos e professores. Não obstante, frequentemente esses aparelhos representam uma preocupação a mais dentro do já difícil contexto educacional do professor. Um dos desafios deste novo milênio, abordado no presente trabalho, é atrelar à prática escolar o uso das novas Tecnologias de Informação e Comunicação, ou TICs.

Diversos resultados promissores do uso de TICs já foram constatados na literatura científica (Balanskat, 2006; Osborne e Hennessy, 2003). Uma pesquisa publicada em 2012 sobre o uso de TICs nas escolas brasileiras revelou que o acesso à internet se encontra disponível para $92 \%$ dos professores

A seção "Educação em Química e Multimídia" tem o objetivo de aprimorar o leitor das aplicações das tecnologias comunicacionais no contexto do ensino-aprendizagem de Química.
Como resultado de nossa experiência com a disciplina Estratégias e Recursos Instrucionais Inovadores no Ensino de Química, ministrada no Mestrado em Ensino de Química da UFRJ (PEQUI), e tendo como público-alvo os professores das escolas públicas, neste trabalho selecionamos alguns recursos instrucionais inovadores que tiveram maior destaque e foram caracterizados conforme suas principais funcionalidades $e$ facilidades para utilização em sala de aula de Química. Esses aplicativos não são direcionados exclusivamente à Química, mas foram avaliados e testados como tendo uso promissor para esse contexto. que lecionam em escolas públicas, e $95 \%$ dos que lecionam em escolas particulares (Cetic, 2012). Conforme investigamos anteriormente, em geral os professores de Química fazem uso frequente das ferramentas disponíveis na internet, porém sua utilização é focada em redes sociais, pesquisa em motores de busca e download de materiais (Rolando et al., 2015). A despeito das grandes inovações não só em termos tecnológicos, mas também pedagógicos, em especial no que concerne às chamadas metodologias ativas de ensino e aprendizado, os professores em sua prática diária ainda utilizam quase exclusivamente os métodos tradicionais de ensino (Bireaud, 1995; Maldaner, 2000; Trevisan e Martins, 2006).

Uma das formas de superar esse desafio é incluir as TICs na formação inicial ou continuada dos professores. No âmbito do ensino da Química, hoje em dia há várias ferramentas direcionadas aos professores que podem ser utilizadas em sala de aula, a exemplo do que foi publicado por Michel et al. (2004) para sítios educacionais. Como resultado de nossa experiência com a disciplina Estratégias 
e Recursos Instrucionais Inovadores no Ensino de Química, ministrada no Mestrado em Ensino de Química da UFRJ (PEQUI), e tendo como público-alvo os professores das escolas públicas, neste trabalho selecionamos alguns recursos instrucionais inovadores que tiveram maior destaque e foram caracterizados conforme suas principais funcionalidades e facilidades para utilização em sala de aula de Química. Esses aplicativos não são direcionados exclusivamente à Química, mas foram avaliados e testados como tendo uso promissor para esse contexto.

\section{Critérios para a seleção}

Os aplicativos foram selecionados tendo professores do Ensino Médio como público alvo, o que exclui diversos aplicativos acadêmicos importantes. Para manter o foco, tomamos como referência os seguintes critérios:

- Facilidade: um aplicativo, para ter ampla aceitação por estudantes e professores, deve ter interface intuitiva e dinâmica, e acesso simples.

- Flexibilidade: o aplicativo deve ser compatível com as principais opções de equipamentos disponíveis em sala, de forma que a existência de diferentes sistemas operacionais não seja um fator limitador.

- Custo zero ou reduzido: considerando a realidade do ensino brasileiro, nenhum dos aplicativos pode ter custo em suas funções essenciais, mantendo, em sua opção gratuita, a capacidade de prover soluções de ensino. Cus- tos adicionais podem ser incluídos para funcionalidades avançadas prescindíveis ao professor.

- Organização: o material deve, preferencialmente, apresentar ferramentas que possibilitem recursos como personalização de materiais, compartilhamento entre usuários, importação e exportação de notas.

Praticamente todos os aplicativos selecionados têm versões disponíveis nos três principais sistemas operacionais, i.e.: Windows, iOS e Android. Aplicativos como blogs e as redes sociais, como o Facebook, não são abordados neste trabalho, uma vez que foram objeto de investigação de diversos autores (Patrício e Gonçalves, 2010; Barro et al., 2008).

Buscando uma classificação geral dos recursos selecionados, foi elaborada uma sequência de legendas de identificação das funcionalidades, conforme a Tabela 1.

\section{Aplicativos selecionados e comentários}

Uma das opções mais funcionais para o professor é o uso de formulários que permitem contribuir para a avaliação metodológica e de aprendizagem. Na Tabela 2 selecionamos os aplicativos que atualmente têm funcionalidades mais favoráveis ao professor.

Todos os formulários de pesquisa oferecem boas opções de personalização, como, por exemplo, inserção de figuras, textos para descrições, diferentes opções de respostas, como múltipla escolha, caixa de texto e verdadeiro ou falso, entre

Tabela 1: Legenda das funcionalidades

\begin{tabular}{|c|c|c|}
\hline Legenda & Sigla & Descrição da função \\
\hline Base de dados & $\mathrm{BD}$ & Armazena planilhas de resultados e atividades criadas/modificadas. \\
\hline $\begin{array}{l}\text { Importação/exportação em formato } \\
\text { texto ou planilha }\end{array}$ & $\mathrm{IE}$ & Importa e/ou exporta arquivos em formato de texto e/ou planilha editável. \\
\hline Interação virtual & IV & $\begin{array}{l}\text { Possibilita a interação entre os participantes, promovendo ambientes de colabo- } \\
\text { ração, trocas e desenvolvimento mútuo. }\end{array}$ \\
\hline Alteração do idioma & Al & Disponibiliza versões em português e/ou espanhol. \\
\hline Personalização de materiais & PM & Personalização de materiais como vídeos, áudio e flips, e sua posterior divulgação. \\
\hline Compartilhamento de materiais & $\mathrm{CM}$ & $\begin{array}{l}\text { Compartilha materiais via ferramentas que permitem trocas entre usuários e, em } \\
\text { alguns casos, não usuários do software. }\end{array}$ \\
\hline Funcionalidades pagas & $\mathrm{FP}$ & $\begin{array}{l}\text { Desenvolve algumas funcionalidades e versões mais avançadas, vinculadas a } \\
\text { pagamento de taxas. }\end{array}$ \\
\hline Extensões móveis & EM & Disponível em aparelhos móveis. \\
\hline
\end{tabular}

Tabela 2: Aplicativos para criação de formulários de pesquisa on-line.

\begin{tabular}{ccc}
\hline Software & Sítio & Funcionalidades disponíveis \\
\hline murveymonkey: & pt.surveymonkey.com & BD IE AI PM CM FP EM \\
Gocrative & www.socrative.com & BD IE IV AI PM CM EM \\
Google forms & www.google.com/forms & BD IE AI PM CM EM \\
\hline
\end{tabular}


outras. Todos os aplicativos disponibilizam, na área exclusiva para o professor, formas de acompanhar o desenvolvimento das respostas, seja de forma geral, como o resultado de uma turma, seja por meio de avaliações individuais.

As três ferramentas possibilitam a utilização de suas funcionalidades em dispositivos móveis. O SurveyMonkey se destaca nesse sentido, por ser o único que exibe ao educador os resultados sob forma de gráficos, sem precisar recorrer à instalação de plug-ins ou à exportação para outros programas. Por outro lado, o SurveyMonkey só permite a criação gratuita de 10 questionários por usuário inscrito. Dessa forma, caso o docente deseje um número maior de materiais, deverá adquirir um pacote pago ou utilizar novos usuários.

Além das funcionalidades usuais, o Formulários Google tem uma característica única e de especial interesse para o ensino, que é a possibilidade de programar a pergunta ao aluno conforme a resposta anterior. Isso o torna um formulário mais interativo, e com possibilidade de aplicação como jogo. O Formulários Google é, de longe, o mais popular entre os professores, e faz parte de um conjunto de aplicativos da Google Inc., como o Google Drive, o Google Agenda e o Google Hangouts. Todos têm muito a contribuir com a dinâmica do ensino, dentro e fora da sala de aula.

Não obstante o potencial dos dois formulários on-line supracitados, o Socrative tem uma vantagem notável: ele pode ser usado para realizar uma votação interativa dinâmica com a turma, na forma de uma corrida. Conforme as respostas corretas são postadas pelos participantes de cada equipe, uma imagem representativa avança em um painel que pode ser projetado em tempo real na sala de aula. Há aplicativos comerciais relativamente caros que são comprados para esse fim; o Socrative tem praticamente a mesma funcionalidade sem custos diretos.

Não faltam outras boas opções de recursos para a criação de questionários on-line, entre as quais destacamos: SurveyGizmo, SurveyTool, Survata, Qualtrics e Questback. Em todos os casos, é possível usar os questionários fora do ambiente dos aplicativos ou do sítio da internet, por meio de um link compartilhável em redes sociais, por exemplo.

Em diversos momentos do processo de ensino de Química é necessário tornar mais visuais conceitos, modelos e representações-chave para o desenvolvimento cognitivo dos alunos acerca dessa ciência. É nessa perspectiva que os aplicativos BKChem.org, ChemSketch e Avogadro se propõem a auxiliar os docentes na construção e representação estrutural de fórmulas químicas e moléculas.
Estes três aplicativos permitem representar estruturalmente moléculas e seus diferentes tipos de ligações, bem como destacar alguns de seus átomos. Além disso, possuem ferramentas de otimização da estrutura criada pelo utilizador e de rotação de moléculas em 3D.

Tanto no BKChem quanto no ChemSketch é possível representar reações químicas; o Avogadro, entretanto, se restringe à estrutura de moléculas. O ChemSketch oferece a facilidade de, ao criar a estrutura molecular, obter sua nomenclatura, fórmula e massa molar. Além disso, é possível copiar a estrutura criada no ChemSketch e colar no Microsoft Word, sem precisar salvar uma versão no software. Caso seja necessária a edição da estrutura inserida no Microsoft Word, basta clicar duas vezes sobre ela: automaticamente, o usuário será redirecionado ao programa de origem.

O ChemSketch e o Avogadro se sobressaem pelas diversas possibilidades de representação molecular, tornando a estética um de seus pontos fortes. O ChemSketch permite trabalhar também com orbitais e interações entre eles, identificação de carbonos quirais e rotações R ou S, além de diversas opções de modelos prontos, como kits de laboratório e estruturas geométricas, e usar a ferramenta de busca para encontrar sítios que apresentem informações sobre a estrutura.

$\mathrm{O}$ aplicativo Avogadro se destaca pela grande variedade de representações estruturais, bem como pelas funcionalidades que podem ser adicionadas, como geometria molecular, cálculo de energia e massa molecular, hibridações, ângulos de ligação, forças de Van der Waals, ligações de hidrogênio e arranjos cristalinos em aglomerados moleculares.

Os três aplicativos cumprem a função de auxiliar o docente na elaboração de fórmulas e estruturas moleculares. Entretanto, o BKChem difere dos demais por apresentar as opções mais básicas, sendo, talvez, de mais fácil utilização por esse motivo.

Os mapas conceituais têm se tornado cada vez mais utilizados como meio para fomentar a apreensão dinâmica do conhecimento (Trindade e Hartwing, 2012). Há inúmeras opções gratuitas e interessantes que permitem ir bem além de conectar textos com setas e balões. Os três recursos citados na Tabela 4 receberam o nosso destaque.

O GoConqr permite acesso apenas pelo sistema Android, ou como plug-in no navegador, e tem a desvantagem de limitar a versão gratuita à criação de apenas 3 disciplinas e 20 recursos. No entanto, apresenta algumas importantes distinções, como os recursos de flashcards, enquetes e notas

Tabela 3: Aplicativos para edição de fórmulas químicas e moléculas.

Software

BKChem.org

क) ChemSketch

A Avogadro
Sítio

Funcionalidades disponíveis

bkchem.zirael.org

Al PM

chemsketch.en.softonic.com

PM

avogadro.softonic.com.br 
Tabela 4: Aplicativos para a criação de mapas conceituais.

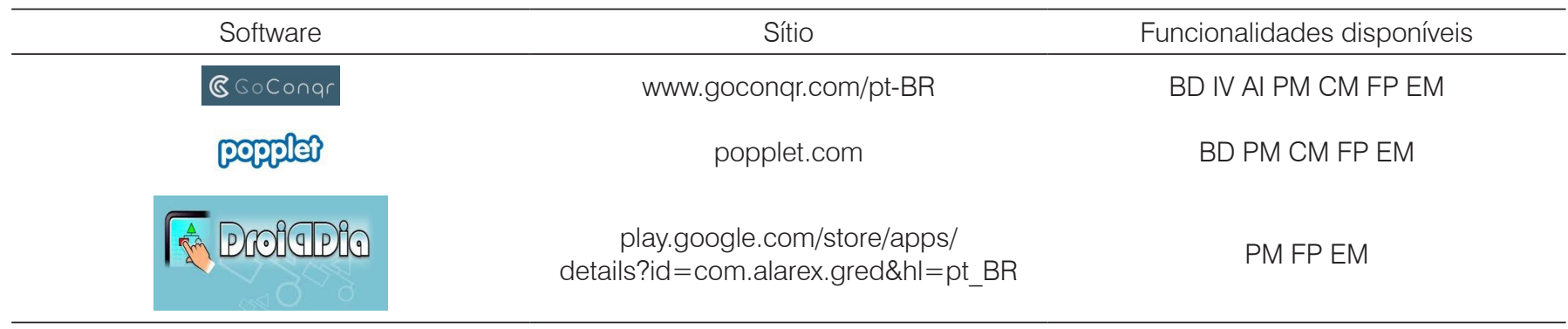

on-line, que podem ser anexadas no mapa. Há ainda a possibilidade de criar grupos e disciplinas para acesso personalizado. O professor pode acompanhar o desenvolvimento da atividade e ter um retorno rápido de seus objetivos.

O DroidDia sobressai pela facilidade de manuseio e funcionalidades disponíveis na versão gratuita, que incluem compartilhamento no aplicativo Whatsapp, e uma grande variedade de materiais que podem ser inseridos nos mapas, incluindo um bom acervo de imagens de sua própria biblioteca. O DroidDia está mais bem adaptado para o uso em tablets, mas funciona bem como plug-in em qualquer computador pessoal.

Outra opção de considerável utilidade é o Popplet. Além de uma interface simples e dinâmica, ideal para os alunos, esse aplicativo prima pela qualidade dos recursos adicionais, como inserção de vídeos (Vimeo e Youtube) e ferramentas de desenho, além da possibilidade de inserir links de outros mapas conceituais. Há muitas opções de compartilhamento e facilidades para o trabalho cooperativo.

Há diversas outras boas opções para elaboração de mapas conceituais, como o SimpleMind,

Free Mind Mapping, Mindly. Vale a pena investigar todas as possibilidades.

Ao longo dos últimos anos, as apresentações em slides vêm ganhando espaço nas propostas metodológicas em sala, representando uma ferramenta auxiliar importante, por vezes indispensável, para melhor visualização de conceitos de Química durante as aulas. O Power Point, do pacote Microsoft Office, embora ainda utilizado em larga escala na produção de apresentações, tem perdido a hegemonia para concorrentes que oferecem funcionalidades semelhantes e mais atraentes, como é o caso do Prezi e Nearpod (Tabela 5).

O grande diferencial do Prezi consiste em sua dinâmica de zoom in e zoom out dentro de um único plano de fundo, usualmente uma imagem, revelando o conteúdo de forma visualmente cativante. Seu funcionamento é intuitivo e a versão gratuita permite um leque amplo de opções para o docente. Além disso, é possível importar arquivos em Power Point para servir como base para as edições, funcionalidade também disponível no Nearpod.

Um dos principais destaques do Nearpod é o recurso de o professor compartilhar as apresentações nos dispositivos dos alunos, sejam tablets ou smartphones. $\mathrm{O}$ docente define a velocidade e a ordem de exibição do material, bem como as páginas a que cada turma terá acesso. Um grande diferencial adicional é que, ao longo das apresentações, é possível adicionar formulários de perguntas com respostas em múltipla escolha para atestar a compreensão dos alunos, o que ainda não é possível no Prezi ou Powerpoint, além de desenhos e pequenos textos. $\mathrm{O}$ professor tem acesso às respostas dos alunos em tempo real em sua plataforma. Entre as limitações do Nearpod estão a capacidade máxima de 30 alunos por turma e o limite de 50MB para armazenamento. Ainda assim, a ferramenta

Tabela 5: Aplicativos para criação de apresentações.

\begin{tabular}{ccc}
\hline Software & Sítio & Funcionalidades disponíveis \\
\hline & prezi.com & BD AI PM CM FP EM \\
& nearpod.com & BD IE \\
\hline
\end{tabular}


recompensa o interesse na dinamização de apresentações e interação com os alunos.

Ponto chave na aprendizagem à distância ou semipresencial, os ambientes virtuais de aprendizagem (AVAs) representam uma forma dinâmica e estruturada de estabelecer novos vínculos de aprendizado. A ferramenta mais utilizada para este fim é o Moodle, amplamente utilizado nas universidades brasileiras, mas praticamente inacessível para o professor das escolas públicas. Diversos outros AVAs mereceriam semelhante destaque, como o Blackboard, e-Proinfo e Teleduc, entre outros. Entretanto, esses AVAs padecem de uma grande burocratização de acesso para a realidade de nossas escolas. O Edmodo, contudo, é uma honrosa exceção (Tabela 6).

Com aparência semelhante ao Facebook, o Edmodo permite a criação de grupos privados, nos quais o docente pode postar mensagens, disponibilizar arquivos importados de diversos formatos, criar formulários de perguntas com diferentes padrões de resposta e fazer enquetes. Além disso, o aplicativo disponibiliza gratuitamente diversas formas de avaliar a atividade realizada pelos alunos e uma área para o acompanhamento do progresso geral.

O Nearpod, apresentado anteriormente, exibe também as características de um AVA, integrando a disponibilização de conteúdo, inserção de atividades, gerência de notas e ambiente colaborativo.

Uma plataforma que, ao longo de sua evolução, tornou-se um AVA, é o Khan Academy, que deveria merecer maior atenção por parte dos professores. Trata-se de um ambiente de aprendizagem que vai muito além de um repositório de vídeos, como muitos costumam supor. Um de seus principais diferenciais é o sistema de autogestão de aprendizado, com dicas para o progresso, em uma interface "viciante", segundo o depoimento dos professores com os quais o testamos na disciplina Estratégias e Recursos Instrucionais Inovadores no Ensino de Química. Até o momento, sua versão traduzida tem como foco a Matemática, que se constitui em um dos principais gargalos no aprendizado de Química. Nossa sugestão é de que o Khan Academy seja utilizado antes de o professor lecionar temas como concentração ou cálculo de $\mathrm{pH}$, como forma de atualização ou como ferramenta diagnóstica.

Jogos e simulações, como na Tabela 7, são outras ferramentas que podem auxiliar o ensino de Química, pela possibilidade de trabalhar conceitos de forma lúdica e interativa.

Nessa perspectiva, o PhET permite não só o trabalho com diversas simulações interativas, a visualização de conceitos abstratos e aspectos microscópicos, como também possibilita ao usuário atuar na variação das condições do sistema em estudo. Nas simulações de Química, é possível encontrar boas opções para trabalhar os conceitos de acidez e basicidade, balanceamento de equações químicas, densidade, polaridade das moléculas, entre outros. Há também várias sugestões de planos de aula baseados nos aplicativos, apesar de nem todas estarem traduzidas para o português. Cabe ressaltar que o professor precisa estar atento à limitação do uso de simulações em função das concepções dos discentes a respeito da escala atômica (Noh e Scharmann, 1997).

Outro recurso meritório é o Banco Internacional de Objetos Educacionais. Organizado pelo Ministério da Educação do Brasil, esse portal oferece acesso gratuito a objetos de aprendizagem em vários formatos e em diversas

Tabela 6: Aplicativos para criação de ambientes virtuais de aprendizado.

Software

Edmodo
Sítio

www.edmodo.com
Funcionalidades disponíveis

BD IE IV AI PM CM EM

Tabela 7: Recursos com jogos e simulações.

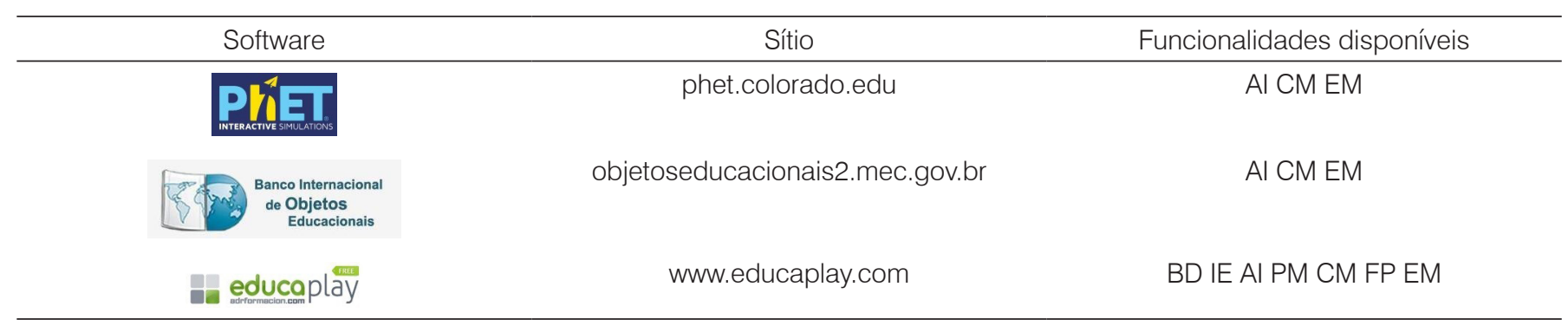


Tabela 8: Aplicativos para o uso de vídeos e edição de áudios.

\begin{tabular}{ccc}
\hline Software & Sítio & Funcionalidades disponíveis \\
\hline EDpuzzle & edpuzzle.com & PM EM IV \\
\hline
\end{tabular}

*Cada aluno pode contribuir na edição de um único vídeo; entretanto, após o envio final, não é possível ao aluno visualizar o material, que ficará disponível somente no perfil do professor.

áreas do conhecimento. Na área de Química, encontram-se disponíveis mais de 1.700 recursos, que incluem áudios, vídeos, animações, imagens, experimentos, simulações e roteiros para aplicação dos recursos em sala de aula.

O Educaplay tem características de um AVA, pois permite a criação de grupos com os alunos, nos quais podem ser disponibilizadas atividades, permitindo ao professor acompanhar o desempenho dos estudantes. Além disso, tem um amplo conjunto de atividades lúdicas já elaboradas por outros educadores e publicadas na plataforma, como adivinhações, ditados, mapa interativo, e quizzes. A ferramenta disponibiliza ainda dicas de como melhorar o material. Vale também consultar o sítio QuiD+ (quid.sbq.org.br).

Hoje em dia, há uma enorme coleção de vídeos que podem ser utilizados como objetos de aprendizagem pelo professor de Química em repositórios, principalmente o Youtube e o Vimeo. Entre os selecionados da tabela 8, o Edpuzzle é uma alternativa eficaz para o docente potencializar o uso educacional dos vídeos. Além de facilitar a edição, com este aplicativo o professor consegue inserir questionários e criar diferentes salas, controlando a quais turmas e atividades os alunos terão acesso. Outro recurso é a de criação de projetos, nos quais os alunos podem escolher e editar vídeos e depois submeter ao docente para análise.

Ainda nessa perspectiva, o aplicativo Audacity se destaca por ser uma ferramenta de gravação e edição de áudios rápida, simples e fácil de manusear, inclusive para arquivos grandes, além de dispor de ferramentas para inserção e personalização de efeitos sonoros e remoção de ruídos. O uso de áudio digital (ou podcasts) como ferramenta didática ainda é modesto no Brasil, mas o potencial é imenso, especialmente em face da possibilidade do discente compreender ou complementar conteúdos fora do contexto da sala de aula, por exemplo, ouvindo no smartphone durante o seu transporte. Essa técnica é conhecida como aula invertida (ou flipped classroom) e, naturalmente, não se aplica apenas aos áudios. O supracitado Banco Internacional de Objetos Educacionais e o Almanaque Sonoro de Química (www.almanaquesonoro. com) são dois repositórios que merecem atenção quanto ao uso didático de podcasts de Química.

\section{Considerações finais}

Os aplicativos selecionados têm características diversificadas e visam cobrir diferentes aspectos das demandas dos professores de Química em sala de aula. Em nossa experiência de emprego e sugestão de uso dessas ferramentas, vencidas as resistências iniciais, em geral o professor sente-se gratificado por este "novo universo". Elas podem ser usadas para a construção de uma aula mais interessante, centrada em uma realidade que produz maior engajamento do aluno, ao mesmo tempo em que pode proporcionar maior suporte para o acompanhamento das atividades escolares. Ademais, com o amadurecimento de seu uso, é necessário menos tempo para o trabalho expositivo em sala de aula, ampliando as opções de novas discussões e atividades. Idealmente, cabe ao docente considerar, em suas aulas, uma perspectiva crítica, criativa e dinâmica em sua proposta metodológica, de forma que as tecnologias não sejam inseridas como meros adereços às aulas, mas que integrem e contribuam para o processo educacional.

Há diversos outros aplicativos que poderiam ser incluídos como foco de interesse para o professor de Química: a seleção aqui apresentada é necessariamente modesta. Além do mais, é preciso destacar que os aplicativos educacionais são dinâmicos, trata-se de recursos com constantes inovações, em uma sociedade ansiosa por novidades.

\footnotetext{
Stephany Petronilho Heidelmann (stephanypheidelmann@ gmail.com.br) é licenciada em Química pelo Instituto Federal de Educação, Ciência e Tecnologia do Rio de Janeiro (IFRJ) e mestranda em Ensino de Química na Universidade Federal do Rio de Janeiro (UFRJ). Rio de Janeiro, RJ - BR. Esteban Lopez Moreno (estebanlmoreno@gmail.com) é professor convidado do Mestrado em Ensino de Química da (PEQUI) na Universidade Federal do Rio de Janeiro (UFRJ) e Professor Associado da Fundação Centro de Ciências e Educação Superior a Distância do Estado do Rio de Janeiro (Fundação CECIERJ). Rio de Janeiro, RJ - BR.
} 


\section{Referências}

BALANSKAT, A.; BLAMIRE, R.; KEFALA, S. The ICT impact report: A review of studies of ICT impact on schools in Europe, 2006.

BARRO, M. R.; FERREIRA, J. Q.; QUEIROZ, S. L. Blogs: Aplicação na Educação em Química. Química Nova na Escola, São Paulo, n. 30, nov. 2008. p. 10-15.

BIREAUD, A. Os métodos pedagógicos no ensino superior. Porto: Porto Editora, 1995.

CETIC. Pesquisa sobre o uso das TIC nas escolas brasileiras: TIC Educação 2012. São Paulo, 2013. Disponível em: www. cetic.br/publicacao/pesquisa-sobre-o-uso-das-tecnologias-de-informacao-e-comunicacao-nas-escolas-brasileiras $/ 88$.

MALDANER, O. A. A formação inicial e continuada de professores de Química. Ijuí: Ed. Unijuí, 2000.

MICHEL, R.; SANTOS, F. M. T.; GRECA, I. M. R. Uma busca na internet por ferramentas para a Educação Química no Ensino Médio. Química Nova na Escola (impresso), São Paulo, v. 19, n. 19, p. 3-7, 2004.

NOH, T.; SCHARMANN, L. C. Instructional influence of a molecular-level pictorial presentation of matter on students' conceptions and problem-solving ability. Journal of Research in Science Teaching. 10.1002/(SICI) 1098-2736(199702)34:2<199::AIDTEA6>3.0.CO;2-O v. 34, i. 2, p. 199-217, February 1997.

OSBORNE, J.; HENNESSY, S., Literature Review in Science
Education and the Role of ICT: Promise, Problems and Future Directions, Bristol: United Kingdom, 2003.

PATRÍCIO, R.; GONÇALVES, V. Facebook: rede social educativa? I Encontro Internacional TIC e Educação. Lisboa: Universidade de Lisboa, Instituto de Educação. p. 593-598, 2010. Disponível em: bibliotecadigital.ipb.pt/bitstream/10198/3584/1/118. pdf. Acesso em: 29 dez. 2015.

ROLANDO, U. G. R.; ROLANDO, R. F. R.; MORENO, E.L.; SALVADOR, D. F.; LUZ, M. R. M. P. Integration between Internet and Chemistry Teaching Practice. Revista Virtual de Química, v. 7, p. 864-879, 2015.

TREVISAN, T. S.; MARTINS, P. L. O. A prática pedagógica do professor de Química: possibilidades e limites. UNIRevista, v. $1, \mathrm{n}^{\circ} 2$, abr. 2006.

TRINDADE, J. O. de, HARTWIG, D. R. Uso combinado de mapas conceituais e estratégias diversificadas de ensino: uma análise inicial das ligações químicas. Química Nova na Escola, São Paulo, v. 34, n. 2, p. 83-91, 2012.

\section{Para saber mais}

Para uma seleção de diversos aplicativos de Química para smartphones, sugerimos a leitura de: LIBMAN, D.; HUANG, L. Chemistry on the go: review of chemistry apps on smartphones. Journal of Chemical Education, v. 90 (3), p. 320-325, 2013. DOI: 10.1021/ed300329e.

Abstract: Innovative instructional resources for teaching chemistry. In this paper we explore some instructional resources for teaching chemistry, especially those which allow teachers to develop a variety of activities with technological support, considering the demands and interests of their students. To select the features covered here, we established criteria such as cost, ease of use and application in the classroom by the teacher. The main features of the elected instructional resources were described.

Keywords: chemistry teaching and learning, computational technologies, chemistry educational applications. 